\title{
Open charm baryon states in the extended local hidden gauge approach
}

\author{
W. H. Liang ${ }^{* \dagger}$ \\ Department of Physics, Guangxi Normal University, Guilin 541004, China \\ E-mail: Liangwheqxnu.edu.cn
}

\section{T. Uchino}

Departamento de Física Teórica and IFIC, Centro Mixto Universidad de Valencia - CSIC, Institutos de Investigación de Paterna, Aptdo. 22085, 46071 Valencia, Spain

\section{W. Xiao}

Institut für Kernphysik, Institute for Advanced Simulation and Jülich Center for Hadron Physics, Forschungszentrum Jülich, 52425 Jülich, Germany

\section{E. Oset}

Departamento de Física Teórica and IFIC, Centro Mixto Universidad de Valencia - CSIC, Institutos de Investigación de Paterna, Aptdo. 22085, 46071 Valencia, Spain

\begin{abstract}
We report on the interaction of $D N, D^{*} N$, together with their coupled channels, by using an extension of the local hidden gauge formalism from the light meson sector, which is based on heavy quark spin symmetry. The scheme is based on the use of the impulse approximation at the quark level, with the heavy quarks acting as spectators, which occurs for the dominant terms where there is the exchange of a light vector meson. In order to perform the full coupled channels calculation, the pion exchange and the Weinberg-Tomozawa interactions are generalized to construct the effective transition potentials. With this dynamics we look for states generated from the interaction, with a unitary coupled channels approach that mixes the pseudoscalar-baryon and vector-baryon states. We find two states with nearly zero width, which are associated to the $\Lambda_{c}(2595)$ and $\Lambda_{c}(2625)$. The lower state, with $J^{P}=1 / 2^{-}$, couples to $D N$ and $D^{*} N$, and the second one, with $J^{P}=3 / 2^{-}$, to $D^{*} N$. In addition to these two $\Lambda_{c}$ states, we find four more states with $I=0$, one of them nearly degenerate in two states of $J=1 / 2,3 / 2$. Furthermore we find three states in $I=1$, two of them degenerate in $J=1 / 2,3 / 2$.
\end{abstract}

XVII International Conference on Hadron Spectroscopy and Structure

25-29 September, 2017

University of Salamanca, Salamanca, Spain

\footnotetext{
* Speaker.

${ }^{\dagger}$ This work is supported by the National Natural Science Foundation of China (Grants No. 11565007, 11647309) and the Spanish Ministerio de Economia y Competitividad and European FEDER funds under the contract number FIS2011-28853-C02-01, FIS2011-28853-C02-02, FIS2014-57026-REDT, FIS2014-51948-C2-1-P, and FIS2014-51948C2-2-P.
} 


\section{Introduction}

In dealing with hadronic states involving heavy quarks (charm or beauty) the heavy quark spin symmetry (HQSS) [W] plays an important role and serves as a guiding principle to proceed with calculations. In Refs. [[], [], it is shown that the Weinberg-Tomozawa term for meson-baryon interaction, which stems from the exchange of vector mesons in the local hidden gauge approach [四, 四, 目], fulfills the HQSS. In Ref. [四], the Weinberg-Tomozawa interaction of the SU(3) sector was extended to the heavy bottom sector and it was shown that the result is equivalent to the plain use of the local hidden gauge approach extended to $\mathrm{SU}(4)$. There the interaction of $B N, \bar{B}^{*} N$, together with their coupled channels, was examined in the local hidden gauge approach, the states $\Lambda_{b}(5912)$ $\left(J^{P}=1 / 2^{-}\right)$and $\Lambda_{b}(5920)\left(J^{P}=3 / 2^{-}\right)$were well reproduced, coupling mostly to $\bar{B}^{*} N$, and the difference of masses comes from the different weight of the intermediate $\bar{B} N$ states, which are accounted for by means of box diagrams mediated by pion exchange. The formalism of Ref. [U] has been used to investigate the meson-baryon interaction in the open charm sector [ [] ] and hidden charm sector $[$ 可].

In the charm sector we have states which bear some similarity with those states in the beauty sector. They are the $\Lambda_{c}(2595)\left(J^{P}=1 / 2^{-}\right)$and $\Lambda_{c}(2625)\left(J^{P}=3 / 2^{-}\right)$states which have been observed in various experiments [ए]]. It is tempting to see if a similar explanation can be given in this case, or see if those states call for a different explanation.

Along the line of Ref. [ $[\mathbb{}]$, here we report on the interaction of $D N, D^{*} N$, together with their coupled channels, investigating the meson-baryon states with open charm by using an extension of the local hidden gauge formalism. We can see that the $\Lambda_{c}(2595)$ and $\Lambda_{c}(2625)$ are generated dynamically from the interaction, with the similarity with the open beauty sector. What differs from the open beauty sector is that, the $\Lambda_{c}(2625)$ state is mostly tied to the $D^{*} N$ channel, while the $\Lambda_{c}(2595)$ state has an important coupling to both the $D N$ and $D^{*} N$ channels. The molecular picture of $\Lambda_{c}(2595)$ and $\Lambda_{c}(2625)$ states has been tested in some decay processes of $\Lambda_{b}$ [प], प2].

\section{Formalism}

For the pseudoscalar-baryon (PB) states we consider the channels $\pi \Sigma_{c}, \pi \Lambda_{c}, \eta \Lambda_{c}, \eta \Sigma_{c}$ and $D N$ which can couple to $I=0,1$; for the vector-baryon $(V B)$ states we have $D^{*} N, \rho \Sigma_{c}, \omega \Lambda_{c}$ and $\phi \Lambda_{c}$ channels. As a singular case, we also consider the single channel $\pi \Sigma_{c}^{*}$, with $\Sigma_{c}^{*}=\Sigma_{c}^{*}(2520)$, belonging to a decuplet of $3 / 2^{+}$states. In the local hidden gauge approach in SU(3) [ [⿴囗十, [1, 团] the meson-baryon interaction proceeds via the exchange of vector mesons as depicted in Fig. $⿴$. In Ref.
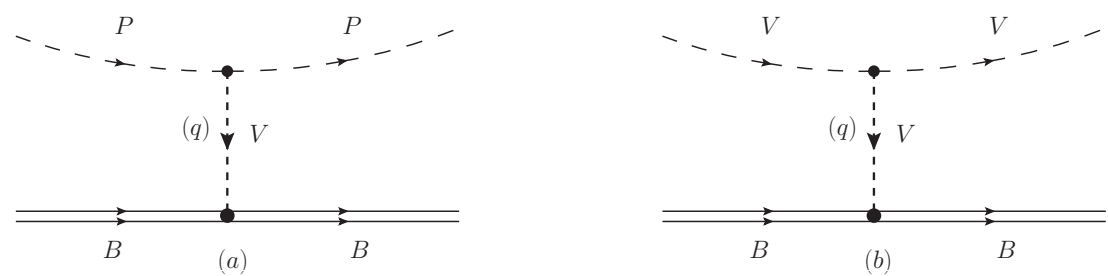

Figure 1: Diagrammatic representation of the pseudoscalar-baryon interaction (a) and vector-baryon interaction (b). 
[ $[$ ], this approach was generalized to SU(4). As discussed in Ref. [प], when we exchange a light vector meson in Figs. W(a) and W(b), the heavy quarks of the meson or the baryon are spectators and, as a consequence, the interaction does not depend on their spin-flavor. Technically, the interaction

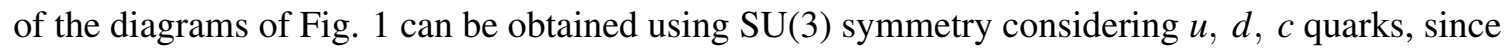
we do not consider states with strangeness or hidden strangeness. Hence, all the matrix elements of the interaction are identical (except for the mass or energy dependence) to those of the interaction of the analogous states $\pi \Sigma, \pi \Lambda, \eta \Lambda, \eta \Sigma, \bar{K} N, \bar{K}^{*} N, \rho \Sigma, \omega \Lambda, \phi \Lambda$ and $\pi \Sigma^{*}$, which have been studied in Refs. [[3], [4]].

For the $P B \rightarrow P B$ and $V B \rightarrow V B$ transitions, the transition potential from channel $i$ to channel $j$ is given by [एᄑ5]

$$
V_{i j}=-C_{i j} \frac{1}{4 f^{2}}\left(2 \sqrt{s}-M_{B_{i}}-M_{B_{j}}\right) \sqrt{\frac{M_{B_{i}}+E_{i}}{2 M_{B_{i}}}} \sqrt{\frac{M_{B_{j}}+E_{j}}{2 M_{B_{j}}}}
$$

with $f=93 \mathrm{MeV}$ the pion decay constant, $M_{B_{i}}, E_{i}\left(M_{B_{j}}, E_{j}\right)$ the mass, energy of baryon of $i(j)$ channel. The $C_{i j}$ coefficients are evaluated in Refs. [지, [4]] and can be found in the Appendix of Ref. [ $[\mathbb{8}]$. The scattering matrix $T$ fulfills the Bethe-Salpeter equation in coupled channels,

$$
T=[1-V G]^{-1} V
$$

with $G$ the diagonal loop function for the propagating intermediate meson-baryon channels. We use here the cut off regularization for $G$ given by

$$
G(s)=\int_{0}^{q_{\max }} \frac{d^{3} \vec{q}}{(2 \pi)^{3}} \frac{\omega_{P}+\omega_{B}}{2 \omega_{P} \omega_{B}} \frac{2 M_{B}}{P^{02}-\left(\omega_{P}+\omega_{B}\right)^{2}+i \varepsilon},
$$

where $P^{0}$ is the CM energy, $s=\left(P^{0}\right)^{2}, \omega_{P}=\sqrt{\vec{q}^{2}+m_{P}^{2}}, \omega_{B}=\sqrt{\vec{q}^{2}+M_{B}^{2}}$, and $q_{\max }$ is the cut-off of the three-momentum.

The mixing of $P B$ and $V B$ states requites $\pi$ exchange, shown in the box diagrams of Fig. \, which involves the $V P \pi$ vertex given by the Lagrangian

$$
\mathscr{L}_{V P P}=-i g\left\langle\left[P, \partial_{\mu} P\right] V^{\mu}\right\rangle,
$$

where $P, V^{\mu}$ are the pseudoscalar and vector matrices, and $g=m_{V} / 2 f$ with $m_{V} \approx 780 \mathrm{MeV}$. It was shown in Ref. [[] that this mixing was responsible for the breaking of the original degeneracy of two spin states of $\Lambda_{b}$, which upon consideration of this mixing became the $\Lambda_{b}(5912)$ and $\Lambda_{b}(5920)$.
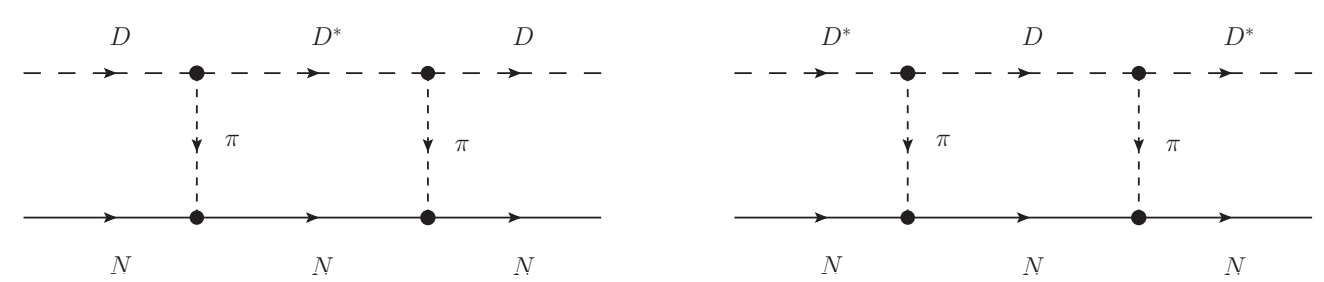

Figure 2: Diagrammatic representation of the $D^{*} N$ in the intermediate state (left) and the $D N$ in the intermediate state (right). 
The evaluation in detail for the contributions of Fig. 凹, $\delta V\left(D N \rightarrow D^{*} N \rightarrow D N\right)$ and $\delta V\left(D^{*} N \rightarrow\right.$ $\left.D N \rightarrow D^{*} N\right)$, can be found in Ref. [8]. Since we only take into account the $P B$ or $V B$ interactions in $s$-wave, in the box diagrams, the intermediate $D^{*} N$ or $D N$ state should be in $s$-wave or $d$-wave for the $J^{P}$ conservation. We need to separate the $s$-wave part from the $d$-wave part of the box diagram. Then we keep the $d$-wave contribution of the box diagram as an additional contribution $\delta V\left(d\right.$-wave) to the $D N \rightarrow D N$ or $D^{*} N \rightarrow D^{*} N$ potentials, and use the $s$-wave part to define the effective transition potential for $D N \rightarrow D^{*} N$ as

$$
V_{D N, D^{*} N}^{\mathrm{eff}}=\frac{1}{2}\left(\tilde{V}_{\mathrm{eff}}+\tilde{V}_{\mathrm{eff}}^{\prime}\right)
$$

with

$$
\begin{aligned}
& \tilde{V}_{\mathrm{eff}}^{2} G_{D^{*} N} \\
& \tilde{V}_{\mathrm{eff}}^{\prime 2} G_{D N} \equiv \delta V\left(D N \rightarrow D^{*} N \rightarrow D N, s \text {-wave }\right), \\
&
\end{aligned}
$$

The expressions for $\delta V\left(D N \rightarrow D^{*} N \rightarrow D N, s(d)\right.$-wave $)$ and $\delta V\left(D^{*} N \rightarrow D N \rightarrow D^{*} N, s(d)\right.$-wave $)$ are shown in Eqs. (31)-(34) of Ref. [8]].

By making the substitutions

$$
\begin{gathered}
V_{D N, D N} \rightarrow V_{D N, D N}+\delta V\left(D N \rightarrow D^{*} N \rightarrow D N, d \text {-wave }\right), \\
V_{D^{*} N, D^{*} N} \rightarrow V_{D^{*} N, D^{*} N}+\delta V\left(D^{*} N \rightarrow D N \rightarrow D^{*} N, d \text {-wave }\right),
\end{gathered}
$$

and adding the $V_{D N, D^{*} N}^{\text {eff }}$ in the Bethe-Salpeter equation Eq. (․2.), we can perform the full coupled channels calculation and look for states generated from the interaction.

\section{Results}

We shall take into account the freedom that we have in the different loops, $P B$ loop, $V B$ loop and box diagram, to take three different cutoffs and adjust them to a few experimental data. In the calculations, we take $q_{\max }^{P}=500 \mathrm{MeV}$ for the cutoff in the $P B$ loop, $q_{\max }^{V}=737 \mathrm{MeV}$ for the cutoff in the $V B$ loop and $q_{\max }^{B}=800 \mathrm{MeV}$ for the cutoff in the box diagrams including the box with the anomalous vertex.

We summarize the final results that we get for meson-baryon states in the charm sector in Table W. We predict six states with $I=0$, two of them corresponding to the $\Lambda_{c}(2595)$ and $\Lambda_{c}(2625)$, and three states with $I=1$, some of them degenerate in spin. The energies of the states range from about $2592 \mathrm{MeV}$ to $3146 \mathrm{MeV}$. It is most instructive to see the nature of the states, which we find by looking both at the couplings $g_{i}$ of the resonance to the different coupled channels, or by looking at the wave functions at the origin $g_{i} G_{i}^{I I}$, with the coupling and $G$ functions evaluated at the pole in the second Riemann sheet. We show this information also in Table $\square$ as the main channel of the state and the main decay channels. From there, we can see that the $\Lambda_{c}(2595)\left(J^{P}=\frac{1}{2}^{-}\right)$couples mostly to $D N$ and $D^{*} N$, and the $\Lambda_{c}(2625)\left(J^{P}=\frac{3}{2}^{-}\right)$couples mostly to $D^{*} N$.

It might seem at first sight that this is a large number of states, but we must recall that for the analogous sector of baryon strange states one finds within the same range of difference of energies 
Table 1: Energies and widths of the states obtained and the channels to which the states couple most strongly. All states have negative parity. Except for the states at $2592 \mathrm{MeV}$ and $2628 \mathrm{MeV}$ that have been fitted to the $\Lambda_{c}(2595)$ and $\Lambda_{c}(2625)$ respectively, and the $\pi \Sigma_{c}^{*}$ at $2674 \mathrm{MeV}$ which is close to the $\pi \Sigma_{c}^{*}$ threshold, the other predicted energies have an estimated uncertainty of $\pm 20 \mathrm{MeV}$.

\begin{tabular}{|c|c|c|c|c|c|}
\hline main channel & $J$ & $I$ & $(E, \Gamma)[\mathrm{MeV}]$ & Exp. & main decay channels \\
\hline$\frac{1}{\sqrt{2}}\left(D N-D^{*} N\right), \pi \Sigma_{c}$ & $1 / 2$ & 0 & 2592,1 & $\Lambda_{c}(2595)$ & $\pi \Sigma_{c}$ \\
\hline$\pi \Sigma_{c}$ & $1 / 2$ & 0 & 2611,106 & - & $\pi \Sigma_{c}$ \\
\hline$\frac{1}{\sqrt{2}}\left(D N+D^{*} N\right)$ & $1 / 2$ & 0 & 2767,2 & - & $\pi \Sigma_{c}$ \\
\hline$D^{*} N$ & $3 / 2$ & 0 & 2628,0 & $\Lambda_{c}(2625)$ & - \\
\hline$\pi \Sigma_{c}^{*}$ & $3 / 2$ & 0 & 2674,111 & - & $\pi \Sigma_{c}^{*}$ \\
\hline$\rho \Sigma_{c}$ & $1 / 2,3 / 2$ & 0 & 2990,2 & $\Lambda_{c}(2940) ?$ & $D^{*} N$ \\
\hline$\pi \Sigma_{c}$ & $1 / 2$ & 1 & 2666,272 & - & $\pi \Sigma_{c}, \pi \Lambda_{c}$ \\
\hline$D^{*} N$ & $1 / 2,3 / 2$ & 1 & 2928,0 & - & - \\
\hline$\rho \Sigma_{c}$ & $1 / 2,3 / 2$ & 1 & 3146,7 & - & $D^{*} N, \rho \Lambda_{c}$ \\
\hline
\end{tabular}

six $\Lambda$ states with spin and parity $J^{P}=1 / 2^{-}, 3 / 2^{-}, 5 / 2^{-}$and six $\Sigma$ states with the same spin and parity, most of which could be reproduced as dynamically generated states of pseudoscalar-baryon or vector-baryon [ए6, [1]].

For the moment there is only one $\Sigma_{c}$ state reported in Ref. [U] ] around $2800 \mathrm{MeV}$. The state, however, has no spin nor parity assigned. While there are several states in Table $\amalg$ close in energy to this state, it is worth quoting that the width of the experimental state is around $75 \mathrm{MeV}$, which is far away from the $\Gamma=0,272 \mathrm{MeV}$, that we find for the likely states in Table $\mathrm{W}$ according to the mass. We would tentatively conclude that the experimental state corresponds most likely to a positive parity state. On the other hand, the reported state $\Lambda_{c}(2940)$ with $\Gamma=17_{-6}^{+8} \mathrm{MeV}$ [एU] which has no spin parity associated, could correspond to the spin degenerate $\Lambda_{c}$ state that we find at $2990 \mathrm{MeV}$ with small width.

\section{Conclusions}

In this work we studied the interaction of $D N$ and $D^{*} N$ states with its coupled channels using dynamics extrapolated from the light quark sector to the heavy one. The starting point was to consider the heavy quarks as spectators in the dominant terms of the interaction. The interaction was extracted mapping from the light sector and respecting the rules of heavy quark spin symmetry, which in the local hidden gauge approach correspond to the exchange of light vectors. The mixing of the $P B$ and $V B$ states was done through pion exchange. This allow us to define the effective transition potential for $D N \rightarrow D^{*} N$. 
With these ingredients, we studied the interaction of the $D N$ and $D^{*} N$ with their coupled channels $\pi \Sigma_{c}, \pi \Lambda_{c}, \eta \Sigma_{c}$ (for the $D N$ ); $\rho \Sigma_{c}, \omega \Lambda_{c}, \phi \Lambda_{c}, \rho \Sigma_{c}^{*}, \omega \Sigma_{c}^{*}, \phi \Sigma_{c}^{*}$ (for the $D^{*} N$ ), and we searched for poles of the scattering matrix in different states of spin and isospin. We found six states in $I=0$, with one of them degenerate in spin $J=1 / 2,3 / 2$, and three states in $I=1$, two of them degenerate in spin $J=1 / 2,3 / 2$. The coupling of the states to the different channels, together with their wave function at the origin, were evaluated to show which is the weight of the different building blocks in those molecular states. In particular, two of the states, one with spin $1 / 2$ that couples mostly to a combination of $D N$ and $D^{*} N$, and a second one with spin $3 / 2$ that couples mostly to $D^{*} N$ were associated to the experimental ones, $\Lambda_{c}(2595)$ and $\Lambda_{c}(2625)$ respectively. The rest of states are so far predictions, with a number of states that is similar to the one of negative parity $\Lambda$ and $\Sigma$ states in the strange sector. In particular we predict another state made of $D N$, $D^{*} N$, orthogonal to the $\Lambda_{c}(2595)$, with a mass around $2767 \mathrm{Mev}$. We think that the use of realistic dynamics, with strict respect of heavy quark spin-flavor symmetry, renders the results obtained rather solid and they should serve as a guideline for future experiments searching for baryon states with open charm.

\section{References}

[1] A.V. Manohar and M.B. Wise. Heavy Quark Physics, Cambridge Monographs on Particle Physics, Nuclear Physics and Cosmology, vol. 10. Camb. Monogr. Part. Phys. Nucl. Phys. Cosmol.10,1

[2] C. W. Xiao, J. Nieves and E. Oset, Phys. Rev. D 88, 056012 (2013).

[3] C. W. Xiao and E. Oset, Eur. Phys. J. A 49, 139 (2013).

[4] M. Bando, T. Kugo, S. Uehara, K. Yamawaki and T. Yanagida, Phys. Rev. Lett. 54, 1215 (1985).

[5] M. Bando, T. Kugo and K. Yamawaki, Phys. Rept. 164, 217 (1988).

[6] U. G. Meissner, Phys. Rept. 161, 213 (1988).

[7] W. H. Liang, C. W. Xiao and E. Oset, Phys. Rev. D 89, 054023 (2014).

[8] W. H. Liang, T. Uchino, C. W. Xiao and E. Oset, Eur. Phys. J. A 51, no. 2, 16 (2015).

[9] T. Uchino, W. H. Liang and E. Oset, Eur. Phys. J. A 52, 43 (2016).

[10] C. Patrignani et al. [Particle Data Group], Chin. Phys. C 40, 100001 (2016).

[11] W. H. Liang, M. Bayar and E. Oset, Eur. Phys. J. C 77, 39 (2017).

[12] W. H. Liang, E. Oset and Z. S. Xie, Phys. Rev. D 95, 014015 (2017).

[13] E. Oset and A. Ramos, Nucl. Phys. A 635, 99 (1998).

[14] S. Sarkar, E. Oset and M. J. Vicente Vacas, Nucl. Phys. A 750, 294 (2005) [Erratum-ibid. A 780, 78 (2006)].

[15] E. Oset, A. Ramos and C. Bennhold, Phys. Lett. B 527, 99 (2002) [Erratum-ibid. B 530, 260 (2002)].

[16] E. Oset, A. Ramos, E. J. Garzon, R. Molina, L. Tolos, C. W. Xiao, J. J. Wu and B. S. Zou, Int. J. Mod. Phys. E 21, 1230011 (2012).

[17] J. A. Oller, E. Oset and A. Ramos, Prog. Part. Nucl. Phys. 45, 157 (2000). 